\title{
Spectroscopic modeling of an argon-doped shock-ignition implosion
}

Cite as: Rev. Sci. Instrum. 81, 10 E307 (2010); https://doi.org/10.1063/1.3479024

Submitted: 25 May 2010. Accepted: 07 July 2010. Published Online: 06 October 2010

R. Florido, R. C. Mancini, T. Nagayama, R. Tommasini, J. A. Delettrez, S. P. Regan, and B. Yaakobi

\section{ARTICLES YOU MAY BE INTERESTED IN}

Investigation of a polychromatic tomography method for the extraction of the threedimensional spatial structure of implosion core plasmas

Physics of Plasmas 19, 082705 (2012); https: / doi.org/10.1063/1.4743017

Time-resolved characterization and energy balance analysis of implosion core in shockignition experiments at OMEGA

Physics of Plasmas 21, 102709 (2014); https://doi.org/10.1063/1.4898329

Understanding reliability and some limitations of the images and spectra reconstructed from a multi-monochromatic x-ray imager

Review of Scientific Instruments 86, 113505 (2015); https://doi.org/10.1063/1.4935828

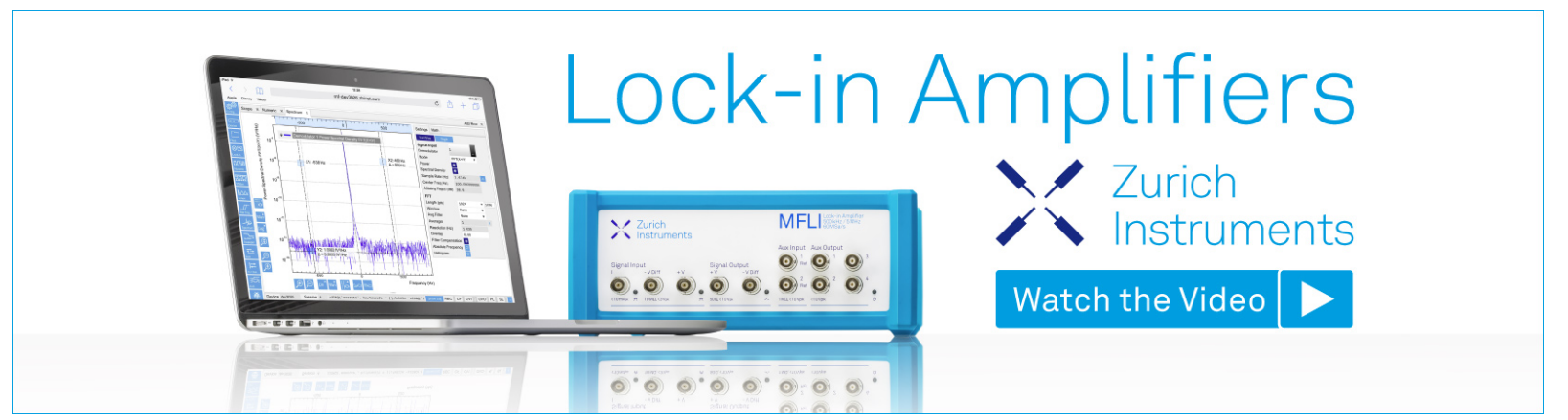

Rev. Sci. Instrum. 81, 10 E307 (2010); https://doi.org/10.1063/1.3479024 


\title{
Spectroscopic modeling of an argon-doped shock-ignition implosion ${ }^{\text {a) }}$
}

\author{
R. Florido, ${ }^{1, b)}$ R. C. Mancini, ${ }^{1}$ T. Nagayama, ${ }^{1}$ R. Tommasini, ${ }^{2}$ J. A. Delettrez, ${ }^{3}$ \\ S. P. Regan, ${ }^{3}$ and B. Yaakobi ${ }^{3}$ \\ ${ }^{1}$ Department of Physics, University of Nevada, Reno, Nevada 89557, USA \\ ${ }^{2}$ Lawrence Livermore National Laboratory, Livermore, California 9450, USA \\ ${ }^{3}$ Laboratory for Laser Energetics, University of Rochester, Rochester, New York 14623, USA
}

(Presented 19 May 2010; received 25 May 2010; accepted 7 July 2010; published online 6 October 2010)

\begin{abstract}
We present results from the spectral postprocessing of a one-dimensional hydrodynamic simulation of an argon-doped, warm-shell shock-ignition implosion with a detailed atomic and radiation physics model. The argon tracer is added to the deuterium filling in the core for diagnostic purposes. Spectral features in the emergent intensity distribution in the photon energy range of the argon K-shell spectrum that have potential for diagnostic application are discussed. () 2010 American Institute of Physics. [doi:10.1063/1.3479024]
\end{abstract}

\section{INTRODUCTION}

Shock-ignition is an approach to inertial confinement fusion $^{1}$ (ICF) that relies on the low-adiabat implosion ${ }^{2}$ of a thick-wall shell target that creates a high areal-density fuel assembly, which is subsequently driven to ignition by a spherically convergent shock wave, i.e., the ignitor shock. ${ }^{3}$ Initial shock-ignition experiments at the OMEGA laser facility ${ }^{4}$ have been successfully performed. ${ }^{5}$ These experiments were diagnosed with particle based diagnostics. Furthermore, shock-ignition has aroused significant interest in the ICF community and several recent studies have been performed for possible shock-ignition experiments at the National Ignition Facility ${ }^{6}$ in the United States as well as in the future European HiPER facility. ${ }^{7}$ In this paper, we discuss the results of postprocessing a one-dimensional (1D) hydrodynamic simulation of an argon-doped, warm-shell shock-ignition implosion, similar to those recently performed at OMEGA, ${ }^{5}$ with a detailed atomic and radiation physics model. The goal is to compute the emergent intensity distribution in the photon energy range of the argon K-shell spectrum in order to investigate spectral signatures that have potential for diagnosing temperature and density in shockignition implosions.

\section{POSTPROCESSING RESULTS}

The target consists of a spherical plastic shell with an initial internal radius of $387 \mu \mathrm{m}$ and a wall thickness of $40 \mu \mathrm{m}$, filled with $20 \mathrm{~atm}$ of $\mathrm{D}_{2}$ and $0.072 \mathrm{~atm}$ of Ar, which is added to the core fill for diagnostic purposes. The laser pulse has a low-adiabat pulse shape of $\sim 18.6 \mathrm{~kJ}$ UV laser energy on target comprised of a 80 ps full width at half maximum (FWHM) picket, followed by a $2 \mathrm{~ns}$ long main

\footnotetext{
${ }^{\text {a) }}$ Contributed paper, published as part of the Proceedings of the 18th Topical Conference on High-Temperature Plasma Diagnostics, Wildwood, New Jersey, May 2010.

${ }^{b)}$ Present address: Departamento de Física, Universidad de Las Palmas de Gran Canaria, 35017 Las Palmas de Gran Canaria, Spain.
}

drive with a sharp intensity spike at the end to launch the ignitor shock wave [see Fig. 1(a)]. These target and laser pulse parameters were selected, in part, based on those used in warm-shell shock-ignition experiments performed at OMEGA. ${ }^{5}$ The 1D Lagrangian hydrodynamics code LILAC (Ref. 8) was used to simulate the implosion. The different stages of the implosion, core boundary, and ablation front locations as well as the effects of the shock waves launched by the laser pulse can be seen in the time-histories of the Lagrangian coordinate trajectories plotted in Fig. 1(b). Maximum compression occurs at $t=4.72$ ns, i.e., approximately $1 \mathrm{~ns}$ after the end of the laser spike, when the core radius reaches a minimum value of $\sim 23 \mu \mathrm{m}$ and the target arealdensity takes a maximum value of $\sim 0.210 \mathrm{~g} \mathrm{~cm}^{-2}$. Figure 2 displays the electron temperature and density spatial profiles in the core and shell at six times during the implosion collapse.

The core's temperatures peak before the core's densities and their spatial profiles are countercorrelated. The range of electron temperatures and densities in the core is suitable for the application of argon K-shell emission as a spectroscopic diagnostic of the implosion. At peak compression, the compressed shell that confines the core reaches electron densities in excess of $1 \times 10^{25} \mathrm{~cm}^{-3}$ with a temperature of about 500 $\mathrm{eV}$. This compressed shell can significantly attenuate the tracer's radiation emitted in the core. We note that the electron number density is related to the mass density via the local average ionization state of the plasma. However, since there is only a tracer amount of argon in the core, the core's electron number density is dominated by the ionization of the deuterium atoms and thus the core's mass density is approximately given by $\rho\left[\mathrm{g} / \mathrm{cm}^{3}\right]=3.2 \times N_{e}\left[10^{24} \mathrm{~cm}^{-3}\right]$ with $3 \%$ accuracy.

The emergent intensity distribution was computed by integrating the radiation transport equation along chords in the target, one photon energy at a time, in the photon energy range relevant for the argon K-shell spectrum, i.e., from 3000 to $4400 \mathrm{eV}$. Then, individual chord contributions were 

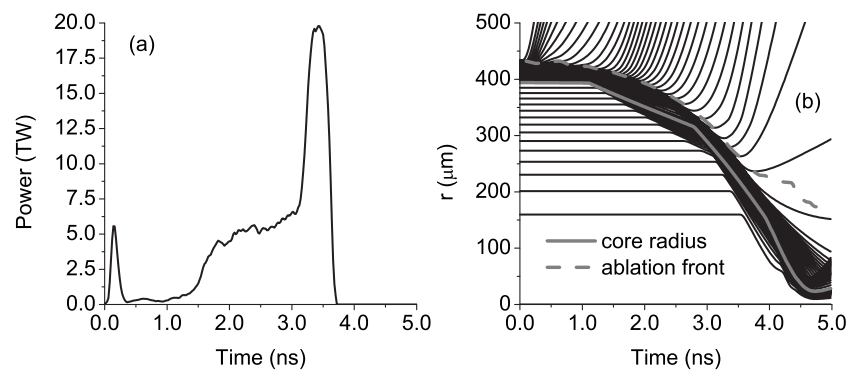

FIG. 1. (a) Laser pulse shape. (b) Time-history of Lagrangian coordinates

further integrated in order to obtain the space-integrated spectrum observed along a line of sight. To this end, the temperature, density, and frequency-dependent emissivity and opacity of the core and shell are required. All boundbound, bound-free, and free-free contributions from the deuterium/argon plasma in the core and the carbon/hydrogen plasma in the shell within the spectral range of interest were included. They were calculated using the atomic level population distributions computed with the collisional-radiative atomic kinetics model ABAKO. ${ }^{9}$ The atomic structure code FAC (Ref. 10) was used to compute energy levels and line transition rates, including unresolved transition arrays ${ }^{11}$ and configuration interaction corrections. ABAKO takes into account all nonautoionizing and autoionizing states consistent with continuum-lowering, which is estimated according to the Stewart-Pyatt model. ${ }^{12}$ Radiation transport effects on level population kinetics were considered via escape factors. ${ }^{13}$ Since Stark-broadened line shapes are important at the densities achieved in the imploded core, a database of line profiles was computed for parent and satellite transitions of the argon K-shell spectrum including the broadening effects due to plasma electron and ion microfields. Furthermore, since deuterium ions are the dominant type of perturbing ion in the plasma core, ion dynamics effects were also considered in the Stark line broadening calculation. ${ }^{14}$ For completeness, natural and Doppler broadening were also included.

The time-history of temperature and density spatial profiles computed by LILAC were postprocessed to obtain spaceintegrated and either time-resolved or time-integrated spectra. From these results the space and photon energy integrated radiation power can be calculated. This result, which is dominated by the argon emission in the core, is shown in Fig. 3(a). The power pulse has a FWHM charac-
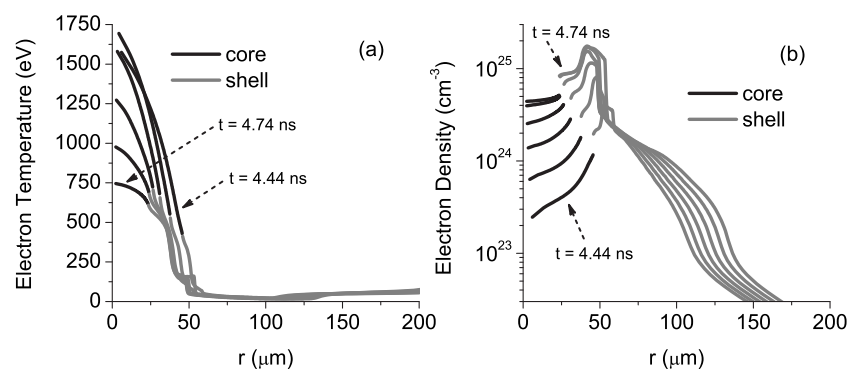

FIG. 2. (a) Core and shell electron temperature and (b) density spatial profiles at six times during the implosion collapse: $t=4.44,4.50,4.56,4.62$, 4.68 , and $4.74 \mathrm{~ns}$.
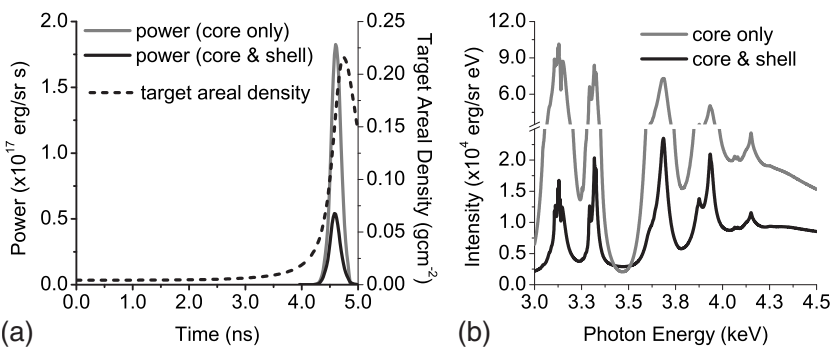

FIG. 3. (a) Time-history of radiation power integrated in the photon energy range from 3000 to $4400 \mathrm{eV}$, including and not including the attenuation effect of the compressed shell and target areal-density. (b) Space-integrated and time-integrated spectrum, including and not including the attenuation effect of the compressed shell.

teristic of $\sim 250 \mathrm{ps}$ and peaks at $t=4.62 \mathrm{~ns}$, before maximum compression. Results are displayed including and not including the attenuation effect of the high areal-density compressed shell, i.e., the attenuation of the radiation emitted by the core as it goes through the compressed shell. This effect is very significant and it is due to bound-free (photoionization) and free-free (inverse bremsstrahlung) absorption transitions in the carbon/hydrogen shell plasma. We note that the cross sections of both atomic processes approximately scale with photon energy as $(h \nu)^{-3}$. As a reference, the timehistory of the target areal-density, which is dominated by the contribution from the compressed shell, is also included in the plot. Next, we consider the time-integrated spectrum shown in Fig. 3(b). Several line transitions of He-like and $\mathrm{H}$-like $\mathrm{Ar}$ ions are observed in the spectrum, namely, $\mathrm{He} \alpha$ $1 s^{2}-1 s 2 p$ at $3140 \mathrm{eV}, \mathrm{Ly} \alpha 1 s-2 p$ at $3320 \mathrm{eV}, \mathrm{He} \beta 1 s^{2}-1 s 3 p$ at $3680 \mathrm{eV}, \mathrm{He} \gamma 1 s^{2}-1 s 4 p$ at $3875 \mathrm{eV}, \mathrm{Ly} \beta 1 s-3 p$ at $3935 \mathrm{eV}$, and $\operatorname{Ly} \gamma 1 s-4 p$ at $4150 \mathrm{eV}$. Weaker, and sometimes heavily blended with parent lines, satellite line transitions arising from autoionizing states in He-like and Li-like Ar ions are also present in the spectrum. Again, we show results including and not including the attenuation through the compressed shell. We can see that the shell's attenuation effect impacts the spectrum in two ways: first, there is an overall reduction in intensity level and second, the attenuation effect is more pronounced at lower photon energies. The latter is consistent with the photon energy dependence of the photoionization and inverse bremsstrahlung cross sections and results in $\mathrm{He} \alpha$ and $\operatorname{Ly} \alpha$ lines that are less intense than the $\operatorname{He} \beta$ and $\operatorname{Ly} \beta$ lines. We emphasize the fact that this shell attenuation effect is in addition to the radiation transport effect on these lines in the core. We note that an attenuation effect due to a high areal-density shell has been previously discussed, but only in connection with continuum emission. ${ }^{15,16}$

In order to understand the formation of the timeintegrated spectrum, Fig. 4 displays time-resolved spectra for six times during the implosion collapse. These are the same six times for which the electron temperature and density spatial profiles are shown in Fig. 2. Time $t=4.62$ ns corresponds to the peak of radiation power emission and time $t$ $=4.74 \mathrm{~ns}$ is just after peak compression. Changes in relative intensity and broadening of the argon lines reflect changes in spatially averaged temperature and density conditions in the core. Early in time, the compressed shell attenuation effect is 

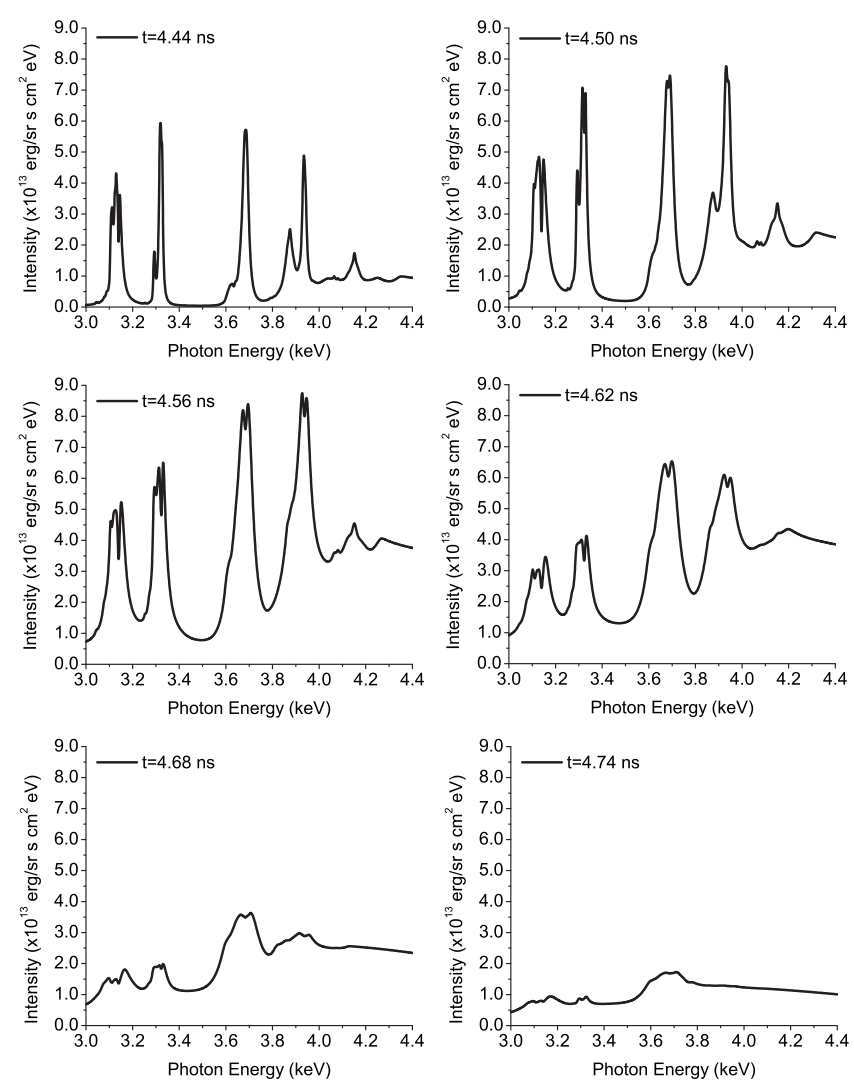

FIG. 4. Synthetic space-integrated, time-resolved spectra for the six electron temperature and density spatial profiles displayed in Fig. 2.

small. But as time progresses and the high areal-density in the compressed shell builds up, this effect becomes more pronounced and significantly knocks down the intensity of both $\alpha$-lines relative to the $\beta$-lines. Late in time, the core cools off, the temperature drops, and the tracer's line emission is gradually lost.

\section{CONCLUSIONS}

We have used a detailed atomic and radiation physics model to postprocess a 1D LILAC hydrodynamic simulation characteristic of an argon-doped, warm-shell shock-ignition implosion at OMEGA. The synthetic spectra in the photon energy range of the argon $\mathrm{K}$-shell emission have signatures that can be employed to diagnose shock-ignition implosions at OMEGA. The spectral features can be recorded in experiments using either a time-integrating or streaked $\mathrm{x}$-ray crystal spectrometer. Core temperature and density conditions are reflected in the intensity distribution of argon line emission. However, the attenuation effect of the high areal-density compressed shell is important and should be taken into account in the spectroscopic analysis. This effect shows up in both time-integrated and time-resolved synthetic spectra. Furthermore, it could be used to extract information about the state of the compressed shell confining the core.

\section{ACKNOWLEDGMENTS}

This work was supported by DOE/NLUF Grant No. DEFG52-09NA29042 and LLNL. R. Florido has also been supported by Spanish Ministry of Science and Innovation Grant No. ENE2008-06668-C02/FTN and the Keep-in-Touch Project of the EU.

${ }^{1}$ J. Lindl, Phys. Plasmas 2, 3933 (1995).

${ }^{2}$ K. Anderson and R. Betti, Phys. Plasmas 11, 5 (2004).

${ }^{3}$ R. Betti, C. D. Zhou, K. S. Anderson, L. J. Perkins, W. Theobald, and A. A. Solodov, Phys. Rev. Lett. 98, 155001 (2007).

${ }^{4}$ T. R. Boehly, D. L. Brown, R. S. Craxton, R. L. Keck, J. P. Knauer, J. H. Kelly, T. J. Kessler, S. A. Kumpan, S. J. Loucks, S. A. Letzring, F. J. Marshall, R. L. McCrory, S. F. B. Morse, W. Seka, J. M. Soures, and C. P. Verdon, Opt. Commun. 133, 495 (1997).

${ }^{5}$ W. Theobald, R. Betti, C. Stoeckl, K. S. Anderson, J. A. Delettrez, V. Y. Glebov, V. N. Goncharov, F. J. Marshall, D. N. Maywar, R. L. McCrory, D. D. Meyerhofer, P. B. Radha, T. C. Sangster, W. Seka, D. Shvarts, V. A. Smalyuk, A. A. Solodov, B. Yaakobi, C. D. Zhou, J. A. Frenje, C. K. Li, F. H. Seguin, R. D. Petrasso, and L. J. Perkins, Phys. Plasmas 15, 056306 (2008).

${ }^{6}$ L. J. Perkins, R. Betti, K. N. LaFortune, and W. H. Williams, Phys. Rev. Lett. 103, 045004 (2009).

${ }^{7}$ X. Ribeyre, G. Schurtz, M. Lafon, S. Galera, and S. Weber, Plasma Phys. Controlled Fusion 51, 015013 (2009).

${ }^{8}$ J. Delettrez, R. Epstein, M. C. Richardson, P. A. Jaanimagi, and B. L. Henke, Phys. Rev. A 36, 3926 (1987).

${ }^{9}$ R. Florido, R. Rodríguez, J. M. Gil, J. G. Rubiano, P. Martel, E. Mínguez, and R. C. Mancini, Phys. Rev. E 80, 056402 (2009).

${ }^{10}$ M. F. Gu, Can. J. Phys. 86, 675 (2008).

${ }^{11} \mathrm{~J}$. Bauche, C. Bauche-Arnoult, and M. Klapisch, Adv. At. Mol. Phys. 23, 131 (1988).

${ }^{12}$ J. C. Stewart and K. D. Pyatt, Astrophys. J. 144, 1203 (1966).

${ }^{13}$ R. C. Mancini, R. F. Joyce, and C. F. Hooper, Jr., J. Phys. B: At. Mol. Phys. 20, 2975 (1987).

${ }^{14}$ R. C. Mancini, D. P. Kilcrease, L. A. Woltz, and C. F. Hooper, Jr., Comput. Phys. Commun. 63, 314 (1991).

${ }^{15}$ B. Yaakobi, R. Epstein, and F. J. Marshall, Phys. Rev. A 44, 8429 (1991).

${ }^{16}$ F. J. Marshall, J. A. Delettrez, R. Epstein, and B. Yaakobi, Phys. Rev. E 49, 4381 (1994). 